www.nature.com/jhg

\title{
Novel PTCH1 mutations in Japanese Nevoid basal cell carcinoma syndrome patients: two familial and three sporadic cases including the first Japanese patient with medulloblastoma
}

\author{
Midori Fujii ${ }^{1}$, Kazuma Noguchi ${ }^{1}$, Masahiro Urade ${ }^{1}$, Yukoh Muraki $^{2}$, Kuniyasu Moridera ${ }^{1}$, \\ Hiromitsu Kishimoto ${ }^{1}$, Tomoko Hashimoto-Tamaoki ${ }^{3,4}$ and Yoshiro Nakano ${ }^{3}$
}

Nevoid basal cell carcinoma syndrome (NBCCS), also known as Gorlin syndrome, is inherited in an autosomal dominant mode, and is characterized by a combination of developmental abnormalities and predisposition to form a variety of tumors. The hedgehog receptor Patched1 (PTCH1) has been identified as the gene mutated in NBCCS. We analyzed PTCH1 in two familial and three sporadic Japanese NBCCS cases, and identified five germline mutations in PTCH1. Two cases have a nonsense mutation (c.3058C $>\mathrm{T}$ and $\mathrm{c.2760C}>\mathrm{A}$ ), one a splice site mutation (c.584+2T $>\mathrm{G}$ ), one a 1 bp insertion (c.2712_2713insA) and one a $1 \mathrm{bp}$ deletion (c.980Gdel). All mutations induce truncation of the PTCH1 protein or could induce nonsense-mediated mRNA decay. The 11-year-old male patient with splice-site mutation (c.584+2T $>$ G) had medulloblastoma (MB) at the age of 1 year. This is the first NBCCS patient with molecularly defined MB in Japan.

Journal of Human Genetics (2011) 56, 277-283; doi:10.1038/jhg.2011.2; published online 3 March 2011

Keywords: Gorlin syndrome; KCOT; medulloblastoma; mutation; Nevoid basal cell carcinoma syndrome (NBCCS); PTCH1

\section{INTRODUCTION}

Nevoid basal cell carcinoma syndrome (NBCCS; MIM 109400), also known as Gorlin syndrome or basal cell nevus syndrome, is a rare autosomal dominant disorder. ${ }^{1,2}$ NBCCS is characterized by developmental defects, such as calcification of the falx celebri, multiple nevi, palmar and plantar pits and skeletal abnormality. ${ }^{2,3}$ It is also known to predispose individuals to cancers, including basal cell carcinoma (BCC), keratocystic odontogenic tumor (KCOT), medulloblastoma $(\mathrm{MB})$, rhabdomyosarcoma and benign tumors, such as ovarian and cardiac fibroma. The hedgehog $(\mathrm{Hh})$ receptor, Patched1 (PTCH1), the human homolog of Drosophila segment polarity gene patched, which is located at chromosome $9 \mathrm{q} 22.3$, has been identified as a gene responsible for NBCCS. ${ }^{4-6}$ PTCH1 spans $34 \mathrm{~kb}$ and consists of at least 23 exons. It encodes a 1447 amino acid protein with 12 potential hydrophobic membrane-spanning domains, intracellular amino-and carboxy-terminal regions and two large hydrophilic extracellular loops in which the Hh protein binds. ${ }^{7,8}$ To date, nearly 300 mutations have been identified in $\mathrm{PTCH} 1 .{ }^{9}$ Among them, more than 150 germline mutations have been reported in NBCCS patients, including nonsense and missense mutations, insertions or deletions and splice site mutations. In addition to NBCCS cases, PTCH1 mutations have also been reported in many malignant neoplasms, such as sporadic BCC, KCOT,
$\mathrm{MB}$, primitive neuroectodermal tumor, breast cancer, colon cancer and meningioma. ${ }^{10,11}$ Nearly $90 \%$ of sporadic BCCs, ${ }^{12} 10-20 \%$ of sporadic $\mathrm{MBs}^{13}$ and approximately $30 \%$ of sporadic KCOTs ${ }^{14}$ carry PTCH1 loss-of-function mutations. KCOT and BCC are two major tumors that characteristically develop in NBCCS patients. However, $\mathrm{MB}$ and other cancers are rarely seen. It is currently unclear whether the locations of mutations within $\mathrm{PTCH} 1$ are relevant to clinical manifestation in NBCCS. However, identification of mutations is very helpful for genetic counseling and for giving clinical advice because patients with mutations are at high risk of NBCCS.

Herein, we identified PTCH1 mutations in two familial and three sporadic NBCCS cases whose first symptoms were KCOTs. One sporadic patient with a splice-site mutation had a history of MB. This is the first report of a molecularly defined Japanese NBCCS patient with MB. In addition, we have analyzed all data from molecularly defined Japanese NBCCS cases. Comparison among ethnically different NBCCS patients will be discussed.

\section{MATERIALS AND METHODS}

All analyses described below were approved by the Medical Ethics Board of Hyogo College of Medicine (No. 108) and Tokuyama Central Hospital. The patients and their parents gave informed consent to participate in this study.

\footnotetext{
${ }^{1}$ Department of Oral and Maxillofacial Surgery, Hyogo College of Medicine, Nishinomiya, Japan; ²Division of Oral Surgery, Tokuyama Central Hospital, Shunan, Yamaguchi, Japan; ${ }^{3}$ Department of Genetics, Hyogo College of Medicine, Nishinomiya, Japan and ${ }^{4}$ Department of Clinical Genetics, Hyogo College of Medicine, Nishinomiya, Japan Correspondence: Dr Y Nakano, Department of Genetics, Hyogo College of Medicine, 1-1 Mukogawa-cho, Nishinomiya, Hyogo 663-8501, Japan. E-mail: y-nakano@hyo-med.ac.jp
}

Received 17 September 2010; revised 25 December 2010; accepted 27 December 2010; published online 3 March 2011 


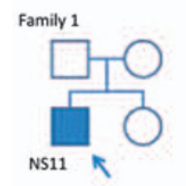

Intron 3 c. $584+2 \mathrm{~T}>\mathrm{G}$

TACAACAGGNAAGGCCCGC
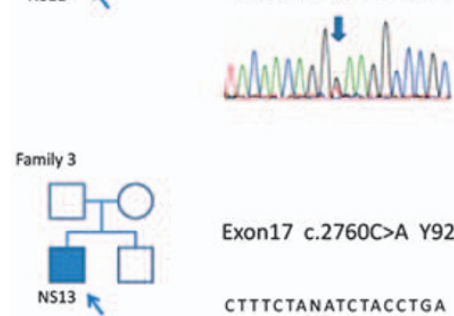

Exon17 c. 2760 C $>$ A Y920X

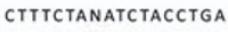

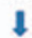

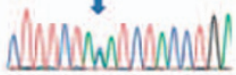

Family 5

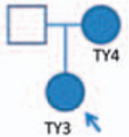

wild-type

Exon7 c.980Gdel 327fsX341

GAATGGTGGATNNNGGNNTANN

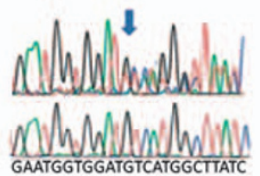

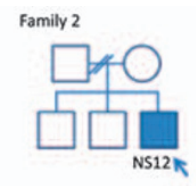

Exon18 c.3058C $>$ T Q1020X

CTGGGAGNAGTACATCG
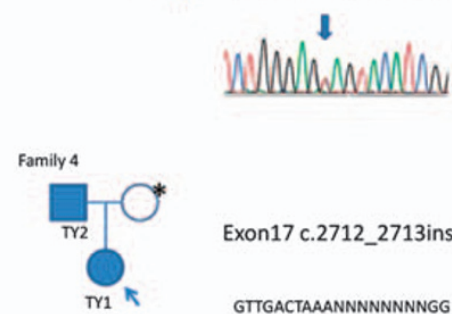

Exon17 c.2712_2713insA 905fsX915

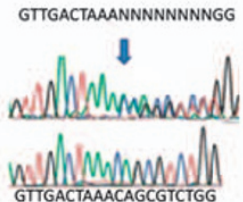

Figure 1 Pedigrees of five families with nevoid basal cell carcinoma syndrome, and electropherograms of the mutations. Filled symbols indicate patients. The arrows indicate the probands. The asterisk $\left({ }^{*}\right)$ represents a healthy individual whose DNA was sequenced.

\section{Patients}

We studied seven Japanese patients from five unrelated families who visited Hyogo College of Medicine or Tokuyama Central Hospital, Japan (Figure 1).

Family 1. An 11-year-old Japanese boy. He was born with congenital cleft lip and palate (Figure 2a), and was treated at the Department of Oral and Maxillofacial Surgery, Hyogo College of Medicine in 1996. During a series of treatments, MB was found (Figure 2c), and surgical resection and chemotherapy were performed. At the age of 11 years, he noticed pus discharge from the right second premolar of the mandible. Panoramic radiography and computed tomography revealed multiple cystic radiolucent areas with impacted teeth (Figure 2b). Moreover, ectopic calcification of the falx cerebri was also detected by computed tomography (Figure 2d). Broad forehead, highly arched eyebrows, ocular hypertelorism and palmar pits were present in this patient. Surgical resection of jaw cysts was performed. Histopathological diagnoses identified all lesions as KCOTs (Figure 2e).

Family 2. A 10-year-old Japanese boy. This patient felt pain in the left, second molar of the mandible, and consulted the Department of Oral and Maxillofacial Surgery, Hyogo College of Medicine. Panoramic radiography and computed tomography revealed multiple cystic radiolucent areas with impacted teeth. He underwent surgical resection of multiple jaw cysts, and histopathological diagnoses identified all lesions as KCOTs. He showed palmar and plantar pits, calcification of the falx cerebri and bifid ribs.

Family 3. A 47-year-old Japanese man. He underwent a series of surgical resections of multiple jaw cysts in the Department of Oral and Maxillofacial Surgery, Hyogo College of Medicine at the age of 36, 38 and 47 years. Histopathological diagnoses identified all lesions as KCOTs. BCCs were found in his skin at the age of 40 years and were surgically removed. He showed palmar and plantar pits, and calcification of the falx cerebri.

Family 4. The proband was a 9-year-old Japanese girl who showed multiple jaw cysts on panoramic radiography. She underwent an operation in the Division of Oral Surgery, Tokuyama Central Hospital. Histopathological diagnoses identified all lesions as KCOTs. She showed palmar and plantar pits and calcification of the falx cerebri. Her father, a 38-year-old Japanese, showed multiple jaw cysts, palmar and plantar pits, calcification of the falx cerebri and macrocephaly. Her mother was healthy.

Family 5. The proband was a 12-year-old Japanese girl who showed multiple jaw cysts on panoramic radiography. She underwent an operation in the Division of Oral Surgery, Tokuyama Central Hospital. Histopathological diagnoses identified all lesions as KCOTs. She showed palmar and plantar pits and calcification of the falx cerebri. Her mother, a 44-year-old Japanese, had KCOTs and showed calcification of the falx cerebri. Her father was healthy.

These cases were diagnosed as having NBCCS by the clinical criteria of Kimonis et al. ${ }^{3}$ (Table 1).

\section{DNA isolation, amplification and sequencing analysis}

Genomic DNA from blood samples was extracted using the QIAamp DNA Blood Midi Kit (Qiagen, Hilden, Germany). All coding exons and intron-exon boundaries were amplified by PCR and sequenced. The primers used in this study have been previously described. ${ }^{15}$ PCR was carried out in a total reaction volume of $50 \mu$ l, containing $100 \mathrm{ng}$ genomic DNA, 1 U Primer STAR HS DNA polymerase (TaKaRa, Ohtsu, Japan), $200 \mu \mathrm{M}$ dNTPs and $0.2 \mu \mathrm{M}$ of each primer. Amplification was carried out for 40 cycles of $98^{\circ} \mathrm{C}$ for $10 \mathrm{~s}, 55^{\circ} \mathrm{C}$ for $5 \mathrm{~s}$ and $72{ }^{\circ} \mathrm{C}$ for $60 \mathrm{~s}$. Amplified products were purified using the MinElute PCR Purification Kit (Qiagen). Sequencing was performed using a BigDye Terminator v3.1 Cycle Sequencing Kit (Applied Biosystems, Foster City, CA, USA) and a 3130xl Genetic Analyzer (Applied Biosystems), according to the manufacturer's instructions.

For analysis of aberrant splicing, total RNA was extracted from cell line, ImNBCCS-KCOT (KCOT-derived cell line from patient NS11: Noguchi K, manuscript in preparation), using the QIAamp RNA Blood Mini Kit (Qiagen) and subjected to RT-PCR using a Cells-to-cDNA II Kit (Applied Biosystems). The forward primer for exon 3 was $5^{\prime}$-TTATACTCGCCAGAAGATTGGAGA AGA- $3^{\prime}$, and the reverse primer for exon 6 was 5'-GTCCATGTAACCATGACCA 

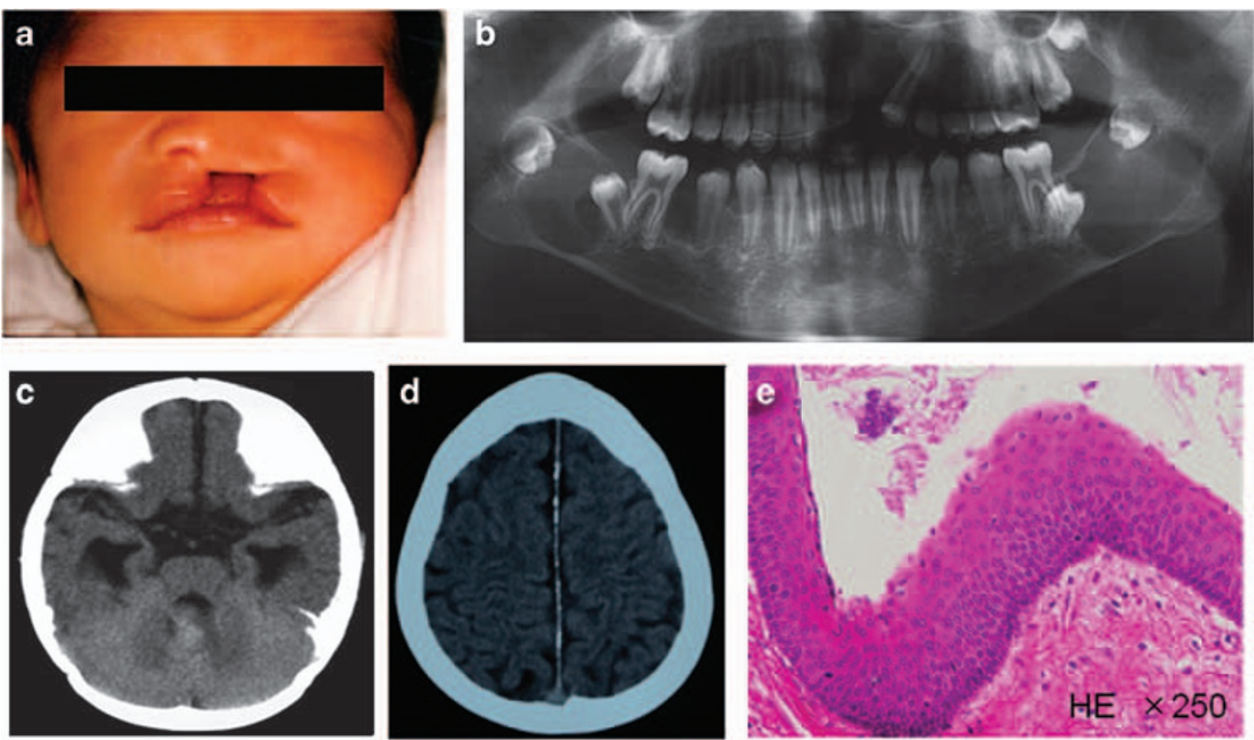

Figure 2 Patient NS11, an 11-year-old boy. (a) Congenital cleft lip and palate. (b) Panoramic radiograph reveals multiple cystic radiolucent areas with impacted teeth (age 11 years). (c) CT showed medulloblastoma in the brain (age 1 years). (d) Ectopic calcification of the falx cerebri (age 11 years). (e) Hematoxylin and eosin (HE) staining shows keratinized squamous epithelium, indicating a keratocystic odontogenic tumor (age 11 years).

Table 1 Characteristics of the patients in this study

\begin{tabular}{|c|c|c|c|c|c|c|c|}
\hline \multirow[b]{2}{*}{ Diagnostic criteria according to Kimonis et al. ${ }^{3}$} & \multicolumn{7}{|c|}{ Patient (age in years) } \\
\hline & NS11 (11) & NS12 (10) & NS13 (47) & $T Y 1$ (9) & TY2 (38) & $T Y 3(12)$ & TY4 (44) \\
\hline \multicolumn{8}{|l|}{ Major symptoms } \\
\hline Basal cell carcinoma (more than two or under the age of 20 years) & - & - & + & - & - & - & - \\
\hline Keratocystic odontogenic tumor & + & + & + & + & + & + & + \\
\hline Palmar and plantar pits (more than three) & + & + & + & + & + & + & - \\
\hline Ectopic calcification of the falx cerebri & + & + & + & + & + & + & + \\
\hline First-degree relative with NBCCS & - & - & - & - & + & - & + \\
\hline \multicolumn{8}{|l|}{ Minor symptoms } \\
\hline Macrocephaly (after correction for height) & - & - & - & - & + & - & - \\
\hline Congenital malformation & + & - & - & - & - & - & - \\
\hline Other skeletal abnormalities & - & - & - & - & - & - & - \\
\hline Abnormality in radiological examination & - & - & - & - & - & - & - \\
\hline Ovarian fibroma & - & - & - & - & - & - & - \\
\hline Medulloblastoma & + & - & - & - & - & - & - \\
\hline
\end{tabular}

ACCTCAG-3'. Amplified products were gel purified and sequenced as described above.

\section{Data analysis of Japanese NBCCS patients}

Data from molecularly defined Japanese NBCCS patients were obtained from published papers. Suspected NBCCS patients, who had not been molecularly defined, were excluded in this analysis. All patients were analyzed based on major diagnostic criteria, such as BCC, KCOT, palmar and plantar pits, calcification of the falx cerebri and various tumors.

\section{RESULTS}

Sequencing analysis revealed $P T C H 1$ mutations in all NBCCS patients (Figure 1, Table 2). The mutations in families 1, 2, 4 and 5 were novel, and the mutation in family 3 was previously reported in a KCOT sample from a Brazilian NBCCS patient. ${ }^{16}$ We found a mutation at a PTCH1 splice donor site in patient NS11, therefore, we analyzed
PTCH1 mRNA using cells derived from a KCOT from NS11. Sequence analysis of DNA derived from KCOT cells and peripheral blood of NS11 showed identical patterns. Therefore, a cell line was derived from the NS11 KCOT and neither additional mutations nor evidence of loss of heterozygosity in PTCH1 were found in these cells. To analyze the splicing events, total RNA was extracted from NS11-derived cells and RT-PCR was performed using a pair of primers located in exon 3 and exon 6 (Figure 3d). RT-PCR produced two bands (Figure 3a), which were sequenced separately (Figures 3b and $\mathrm{c}$ ). The short transcript, transcript 1 , consisted of wild-type sequences (Figure $3 \mathrm{~b}$ ). The longer fragment revealed that this mutation activated a cryptic splice site in intron 3 , resulting in a $37 \mathrm{bp}$ insertion of intronic sequence into the mRNA (Figures $3 \mathrm{c}$ and $\mathrm{d}$ ). This insertion induced a frame shift and termination after 58 additional amino acids. This additional sequence did not show obvious homology to any other known protein. 
In families 2 and 3, the mutations created stop codons (Table 2). In families 4 and 5, the mutations caused frame shifts, resulting in stop codons after 11 and 15 amino acids, respectively.

The spectrum of PTCH1 mutations in Japanese NBCCS patients was analyzed based on the literature. ${ }^{15,17-28}$ Table 3 summarizes the spectrum of PTCH1 mutations and phenotypes of Japanese NBCCS

Table 2 Germline mutations in the PTCH1 gene

\begin{tabular}{llll}
\hline Family & Exon & Mutation $^{\mathrm{a}}$ & Effect on protein \\
\hline 1 & Intron 3 & c.584+2T $>\mathrm{G}$ & $\begin{array}{l}\text { Insertions and frameshift } \\
\text { p.Q1020X }\end{array}$ \\
2 & Exon 18 & c.3058C $>\mathrm{T}$ & p.Y920X \\
3 & Exon 17 & c.2760C $>$ A & p.R905fsX915 \\
4 & Exon 17 & c.2712_2713insA & p.R327fsX341 \\
5 & Exon 7 & c.980Gdel & \\
\hline aNM_000264. & & & \\
bNP_000255. & & &
\end{tabular}

patients. Overall 56 subjects who fulfilled the criteria for NBCCS were analyzed. There were 31 men and 25 women, ranging in age from 0 to 71 years (average 26.3 years). Mutations were scattered throughout the entire gene. Therefore, there is no mutational hot spot and also no founder effect for NBCCS in Japan. Patients with KCOTs represented $87.3 \%(48 / 55)$ of patients. Pits were observed in $43 / 53$ $(81.1 \%)$ of cases. Calcification of the falx cerebri was seen in $39 / 50$ (78.0\%) of cases. BCC occurred in 14/56 (25.0\%) of cases. 8/15 (53.3\%) of cases over 40 years of age had developed a BCC. Other cancers were reported in two cases, one $\mathrm{MB}$ (our study) and one meningioma.

\section{DISCUSSION}

We analyzed two familial and three sporadic Japanese NBCCS patients, and identified germline $P T C H 1$ mutations in all patients: one splice site mutation $(c .584+2 \mathrm{~T}>\mathrm{G})$, two nonsense mutations (c.3058C $>$ T and c.2760C $>$ A), one 1 bp insertion (c.2712_2713insA) and one $1 \mathrm{bp}$ deletion (c.980Gdel). All mutations induce premature

a

b

EXON 3

EXON 4
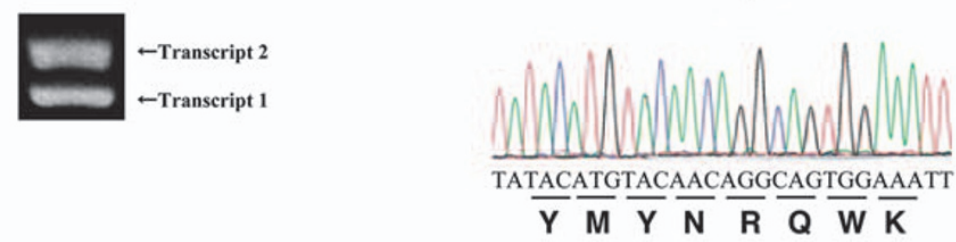

C EXON 3

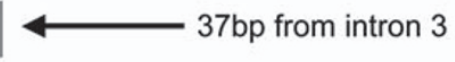
EXON 4

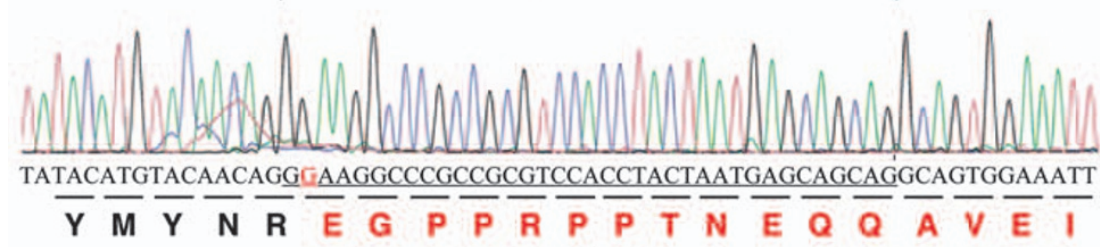

d Transcript 1

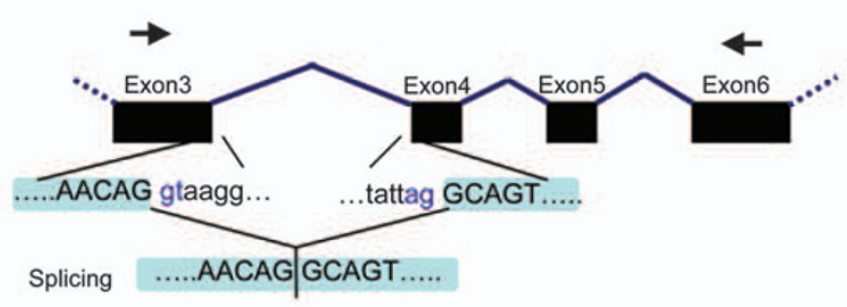

Transcript 2

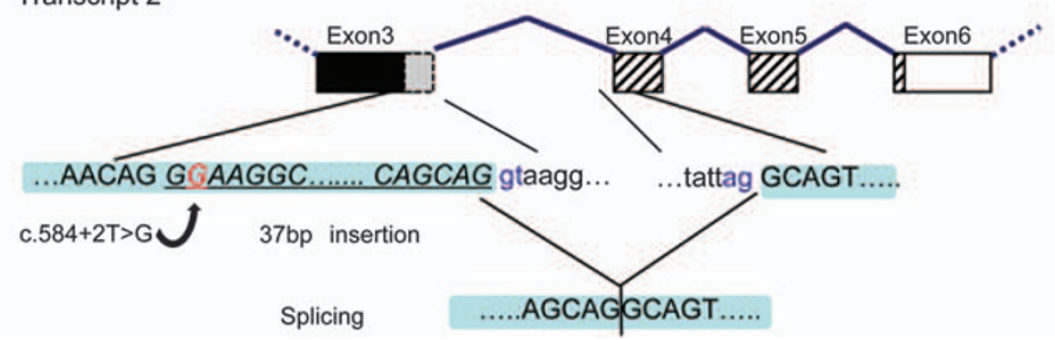

Figure 3 The PTCH1 mutation found in patient NS11 (c.584+2T>G) induced aberrant splicing. (a) Analysis of the splice donor mutation in NS11. RT-PCR was carried out using RNAs from NS11-derived cells. Primers are illustrated as black arrows in (d). Gel electrophoresis of RT-PCR products produces two bands. (b, c) Sequence analysis of the two RT-PCR products. Lower fragment (transcript 1) indicated a normal splicing event (b). The upper fragment (transcript 2) showed that the c.584+2T $>$ G mutation led to the activation of a cryptic splice site in intron 3 , resulting in the insertion of a $37 \mathrm{bp}$ intronic sequence into the mRNA (c). (d) Schematic representation of the splicing events induced by the $c .584+2 T>G$ mutation. One allele produced normal mRNAs (transcript 1). The other mutated allele produced mRNAs with a $37 \mathrm{bp}$ insertion resulting in a frame shift mutation. Black boxes indicate coding exons. Gray box indicates intronic sequence inserted into the mRNA. Hatched areas show frame-shifted regions. White represents non-coding sequence. 
Table 3 PTCH1 mutations and clinical features of Japanese nevoid basal cell carcinoma syndrome patients

\begin{tabular}{|c|c|c|c|c|c|c|c|c|c|c|}
\hline Type of mutation & Exon & Nucleotide change & $\begin{array}{c}\text { Age } \\
\text { (in years) }\end{array}$ & Sex & $B C C$ & KCOT & Pits & $\begin{array}{l}\text { Calcification of } \\
\text { falx cerebri }\end{array}$ & Other cancer & Reference \\
\hline 2 bp deletion & 2 & c.239_240delGA & 18 & M & - & + & + & + & - & Takahashi et al. ${ }^{15}$ \\
\hline Nonsense & 2 & c. $279 \mathrm{C}>\mathrm{A}$ & 16 & $\mathrm{~F}$ & - & + & + & + & - & Minami et al. ${ }^{17}$ \\
\hline Missense & 2 & c. $328 \mathrm{G}>\mathrm{T}$ & 19 & M & - & + & + & + & - & Tanioka et al. ${ }^{22}$ \\
\hline Missense & 2 & c. $328 \mathrm{G}>\mathrm{T}$ & 43 & $\mathrm{~F}$ & - & + & + & - & - & Tanioka et al. ${ }^{22}$ \\
\hline $1 \mathrm{bp}$ deletion & 3 & c.459delT & 27 & $\mathrm{~F}$ & + & + & + & + & - & Nagao et al. ${ }^{21}$ \\
\hline Splicing & 3 & c. $584 \mathrm{G}>\mathrm{A}$ & 61 & M & + & + & + & - & - & Nagao et al. ${ }^{21}$ \\
\hline Splicing & intron 3 & c. $584+2 \mathrm{~T}>\mathrm{G}$ & 11 & M & - & + & + & + & Medulloblastoma & This study \\
\hline 1 bp deletion & 6 & c.865delC & 29 & M & - & + & + & + & - & Tanioka et al. ${ }^{22}$ \\
\hline 1 bp deletion & 6 & c.912delC & 18 & $\mathrm{~F}$ & - & + & + & + & - & Fujii et al. ${ }^{19}$ \\
\hline 1 bp deletion & 6 & c.912delC & 16 & $\mathrm{~F}$ & - & + & + & + & - & Fujii et al. ${ }^{19}$ \\
\hline Splicing & intron6 & c. $945+5 G>T$ & 5 & M & - & - & + & - & - & Fujii et al. ${ }^{19}$ \\
\hline 1 bp deletion & 7 & c.980Gdel & 12 & $\mathrm{~F}$ & - & + & + & + & - & This study \\
\hline 1 bp deletion & 7 & c.980Gdel & 44 & $\mathrm{~F}$ & - & + & - & + & - & This study \\
\hline Splicing & intron7 & c. $1067+1 G>C$ & 16 & M & - & + & - & + & - & Sasaki et al. ${ }^{27}$ \\
\hline $58 \mathrm{bp}$ duplication & 8 & c.1138_1195dup & 12 & M & - & + & + & + & - & Takahashi et al. ${ }^{15}$ \\
\hline Missense & 8 & c. $1162 A>T$ & 70 & $\mathrm{~F}$ & + & - & NR & NR & - & Ogata et al. ${ }^{18}$ \\
\hline Nonsense & 8 & c. $1196 \mathrm{G}>\mathrm{A}$ & 10 & $\mathrm{~F}$ & - & + & - & + & - & Takahashi et al. ${ }^{15}$ \\
\hline Nonsense & 8 & c. $1196 \mathrm{G}>\mathrm{A}$ & 0 & $\mathrm{~F}$ & - & - & - & NR & - & Takahashi et al. ${ }^{15}$ \\
\hline 1 bp insertion & 9 & c.1261insT & 17 & $\mathrm{~F}$ & - & + & + & + & - & Fujii et al. ${ }^{19}$ \\
\hline 4 bp duplication & 10 & c.1416_1419dupTGGC & 3 & M & - & - & + & + & - & $\begin{array}{l}\text { Nagao et al. }{ }^{21} \text {, Tachi et al. }{ }^{24} \text {, } \\
\text { Honma et al.. }\end{array}$ \\
\hline 1 bp deletion & 10 & 1427delT & 15 & $\mathrm{~F}$ & - & + & + & + & - & Sasaki et al. ${ }^{28}$ \\
\hline Missense & 11 & c. $1526 \mathrm{G}>\mathrm{A}$ & 66 & M & + & + & + & + & - & Fujii et al. ${ }^{19}$ \\
\hline Missense & 12 & c. $1660 A>C$ & 44 & M & - & + & - & + & - & Tanioka et al. ${ }^{22}$ \\
\hline 2 bp deletion & 12 & c.1670_1671delCA & 18 & $\mathrm{~F}$ & - & + & + & + & - & Takahashi et al. ${ }^{15}$ \\
\hline $2 \mathrm{bp}$ deletion & 12 & c.1670_1671delCA & 46 & $\mathrm{~F}$ & - & + & + & + & - & Takahashi et al. ${ }^{15}$ \\
\hline 1 bp deletion & 14 & c.2011delC & 59 & $\mathrm{~F}$ & + & + & + & + & - & Fujii et al. ${ }^{19}$ \\
\hline 1 bp deletion & 14 & c.2029delA & 23 & $\mathrm{~F}$ & - & + & + & + & - & Takahashi et al. ${ }^{15}$ \\
\hline 1 bp deletion & 15 & c.2395delT & 29 & M & + & + & + & + & - & Minami et al. ${ }^{17}$ \\
\hline 1 bp deletion & 15 & c.2395delT & NR & M & + & + & - & + & - & Minami et al. ${ }^{17}$ \\
\hline 1 bp deletion & 15 & c.2395delT & NR & M & + & + & - & + & - & Minami et al. ${ }^{17}$ \\
\hline 1 bp duplication & 15 & c.2454dupA & 28 & M & - & + & + & + & - & Tanioka et al. ${ }^{22}$ \\
\hline 1 bp deletion & 16 & c.2613delC & 50 & $\mathrm{~F}$ & + & + & NR & NR & Meningioma & Tate et al. ${ }^{20}$ \\
\hline 1 bp deletion & 16 & c. 2613 delC & NR & $\mathrm{F}$ & + & NR & NR & NR & - & Tate et al. ${ }^{20}$ \\
\hline 1 bp insertion & 17 & c.2712_2713insA & 9 & $\mathrm{~F}$ & - & + & + & + & - & This study \\
\hline 1 bp insertion & 17 & c.2712_2713insA & 38 & M & - & + & + & + & - & This study \\
\hline 1 bp insertion & 17 & c.2724_2725insT & 26 & $\mathrm{~F}$ & - & + & + & + & - & Nagao et al. ${ }^{21}$ \\
\hline Missense & 17 & c. $2760 \mathrm{C}>\mathrm{A}$ & 40 & $\mathrm{M}$ & + & + & + & + & - & This study \\
\hline $\begin{array}{l}22 \text { bp deletion and } \\
9 \text { bp insertion }\end{array}$ & 17 & c.2785_2806del22ins9 & 20 & M & - & + & + & + & - & Tanioka et al. ${ }^{22}$ \\
\hline Nonsense & 18 & c. $2908 \mathrm{G}>\mathrm{T}$ & 71 & M & + & - & + & + & - & Tanioka et al. ${ }^{22}$ \\
\hline 1 bp deletion & 18 & c.3016delC & 46 & $\mathrm{~F}$ & + & + & + & - & - & Tanioka et al. ${ }^{22}$ \\
\hline Missense & 18 & c. $3058 \mathrm{C}>\mathrm{T}$ & 10 & M & - & + & + & + & - & This study \\
\hline 2 bp duplication & 18 & c.3130_3131dupGC & 3 & $\mathrm{M}$ & - & + & + & - & - & Nagao et al. ${ }^{21}$ \\
\hline 2 bp duplication & 18 & c.3130_3131dupGC & 37 & $\mathrm{~F}$ & - & + & + & - & - & Nagao et al. ${ }^{21}$ \\
\hline 4 bp duplication & 20 & c.3325_3328dupGGCG & 23 & M & - & + & + & + & - & Sasaki et al. ${ }^{27}$ \\
\hline 2 bp deletion & 20 & c.3364_3365 delAT & 10 & M & - & + & + & - & - & Nagao et al. ${ }^{21}$ \\
\hline Missense & 20 & c. $3398 \mathrm{C}>\mathrm{T}$ & 47 & $\mathrm{~F}$ & - & + & + & + & - & Otsubo et al. ${ }^{26}$ \\
\hline Missense & 20 & c. $3398 \mathrm{C}>\mathrm{T}$ & 22 & M & - & + & + & + & - & Otsubo et al. ${ }^{26}$ \\
\hline Missense & 20 & c. $3398 \mathrm{C}>\mathrm{T}$ & 19 & $\mathrm{~F}$ & - & + & + & + & - & Otsubo et al. ${ }^{26}$ \\
\hline Missense & 20 & c. $3398 \mathrm{C}>\mathrm{T}$ & 17 & $\mathrm{M}$ & - & + & + & - & - & Otsubo et al. ${ }^{26}$ \\
\hline $165 \mathrm{~kb}$ deletion & $1-23$ & & 10 & M & - & + & - & - & - & Fujii et al. ${ }^{23}$ \\
\hline $165 \mathrm{~kb}$ deletion & $1-23$ & & 43 & $\mathrm{~F}$ & - & - & - & - & - & Fujii et al. ${ }^{23}$ \\
\hline 1.2 Mb deletion & $1-23$ & & 12 & M & - & + & + & + & - & Takahashi et al. ${ }^{15}$ \\
\hline 1.2 $\mathrm{Mb}$ deletion & $1-23$ & & 40 & M & - & + & + & NR & - & Takahashi et al. ${ }^{15}$ \\
\hline 1.2 Mb deletion & $1-23$ & & 8 & M & - & + & + & NR & - & Takahashi et al. ${ }^{15}$ \\
\hline 5.3 Mb deletion & $1-23$ & & 8 & M & - & - & + & - & - & Fujii et al. ${ }^{23}$ \\
\hline \multirow[t]{3}{*}{$11 \mathrm{Mb}$ deletion } & $1-23$ & & 12 & $\mathrm{M}$ & + & + & - & + & - & Fujii et al. ${ }^{23}$ \\
\hline & & & ave. & $\mathrm{M}: \mathrm{F}$ & $14 / 56$ & 48/55 & $43 / 53$ & $39 / 50$ & $2 / 55$ & \\
\hline & & & 26.3 & $31: 25$ & $25.0 \%$ & $87.3 \%$ & $81.1 \%$ & $78.0 \%$ & $3.6 \%$ & \\
\hline
\end{tabular}

Abbreviations: ave., average; BCC, basal cell carcinoma; F, female; KCOT, keratocystic odontogenic tumor; M, male; NR, not reported. 
Table 4 Comparison of frequency of major criteria in NBCCS patients among seven studies

\begin{tabular}{|c|c|c|c|c|c|c|c|}
\hline & $\begin{array}{c}\text { UK; } \\
\text { Evans et al.29 }\end{array}$ & $\begin{array}{c}\text { Australia; } \\
\text { Shanley et al. }\end{array}$ & $\begin{array}{c}\text { USA; } \\
\text { Kimonis et al. }{ }^{3}\end{array}$ & $\begin{array}{c}\text { Italy; } \\
\text { Lo Muzio et al. }{ }^{31}\end{array}$ & $\begin{array}{c}\text { Korea; } \\
\text { Ahn et al. }{ }^{32}\end{array}$ & $\begin{array}{c}\text { Iran; } \\
\text { Habibi et al. }{ }^{33}\end{array}$ & $\begin{array}{c}\text { Japan; } \\
\text { this study }\end{array}$ \\
\hline Patients & 84 & 118 & 105 & 37 & 33 & 19 & 56 \\
\hline Male/female & NR & $51: 67$ & $48: 57$ & $16: 21$ & $16: 17$ & $10: 9$ & $31: 25$ \\
\hline \multicolumn{8}{|c|}{ Major criteria number (\%) } \\
\hline$>20$ years & $33 / 45(73)$ & $71 / 84(85)$ & $58 / 64(91)^{a}$ & $2 / 25(8)^{c}$ & NR & $5 / 10(50)$ & $10 / 26(38$ \\
\hline$>40$ years & $19 / 21(90)$ & $35 / 37(95)$ & $34 / 35(97)^{a}$ & $9 / 12(75)$ & NR & $2 / 4(50)$ & $8 / 15(53$ \\
\hline KCOTs & $46 / 70(66)$ & $85 / 113(75)$ & $78 / 105(74)$ & $34 / 37(92)$ & $30 / 33(91)$ & 19/19 (100) & $48 / 55(87$ \\
\hline$>20$ years & $37 / 45(82)$ & $66 / 82(80)$ & $60 / 74(81)$ & NR & NR & $10 / 10(100)$ & $21 / 26(81$ \\
\hline$>40$ years & $19 / 21(90)$ & $25 / 35(71)$ & $29 / 38(76)$ & NR & NR & $4 / 4(100)$ & $11 / 15(73$ \\
\hline Pits & $50 / 70(71)$ & $82 / 103(80)$ & $89 / 102(87)$ & $13 / 37(35)$ & $22 / 33(67)$ & $14 / 19(74)$ & $43 / 53(81$ \\
\hline
\end{tabular}

Abbreviations: BCC, basal cell carcinoma; KCOT, keratocystic odontogenic tumor; NR, not reported.

awite.

${ }^{\mathrm{b}}$ African American.

${ }^{\mathrm{c}} \mathrm{O}-40$ years.

termination of the PTCH1 protein or could induce nonsensemediated mRNA decay.

A point mutation in intron $3($ c. $.584+2 \mathrm{~T}>\mathrm{G})$ of $P$ TCH1 was detected in patient NS11. RT-PCR analysis revealed that at least two transcripts were generated from this region (Figure 3a). Sequence analysis indicated that the short transcript, transcript 1 , consisted of wild-type sequences (Figure $3 \mathrm{~b}$ ), whereas the long transcript, transcript 2, consisted of a mutated sequence (Figure 3c). These data suggested that the long transcript was not the product of a normal alternative splicing event and that c. $584+2 \mathrm{~T}>\mathrm{G}$ generated a cryptic splice site in intron 3, resulting in the insertion of 37 extra bases into the mRNA. This activated splice site conformed to the authentic GT-AG rule. Interestingly, a very similar, but not identical mutation, which generates an identical 37-bp insertion, has been reported. ${ }^{21}$ In that case, the mutation, c.584G $>A$, was in the exon, but affected the splice donor sequence and induced aberrant splicing. The cryptic splice site generated by c. $584 \mathrm{G}>\mathrm{A}$ was identical to that generated by c. $584+2 \mathrm{~T}>\mathrm{G}$.

Patient NS11 showed a complicated cleft lip and palate with MB (Figure 2). In NBCCS, the morbidity rate of cleft lip and palate is approximately $5 \%$, which is the same as that of MB. ${ }^{3,29,30}$ Therefore, this is a very rare case. His clinical manifestation was severe, but none of his family exhibited NBCCS symptoms; therefore, this mutation seems to be de novo. This is the first report of a Japanese NBCCS patient with MB. PTCH1 mutations in sporadic MBs are scattered throughout the entire gene, ${ }^{9}$ similar to that of NBCCS cases, indicating no correlation between the position of the mutations and the observed phenotype. His KCOT-derived cells showed neither extra mutations nor evidence of loss of heterozygosity, at least in the PTCH1-coding region. Therefore, additional alterations, in addition to that in observed PTCH1, must be involved in this KCOT formation.

Clinical manifestations in NBCCS individuals have been reported in different ethnic groups. ${ }^{3,29-33}$ Comparison of the frequencies of major phenotypes in NBCCS, such as BCCs, KCOTs, palmar or plantar pits and calcification of the falx cerebri are listed in Table 4. Obvious differences were found in the onset of BCCs. In Caucasian NBCCS patients, more than $70 \%$ (over the age of 20 years) or $90 \%$ (over the age of 40 years) of patients developed BCC. ${ }^{3,29,30}$ Kimonis et al. ${ }^{3}$ estimated that the probability of developing BCCs was approximately
$90 \%$ in Caucasians and $40 \%$ in African-Americans by the age of 35 years. In contrast, BCCs developed in only $38 \%$ (over the age of 20 years) and $53 \%$ (over the age of 40 years) of Japanese patients. On the other hand, KCOT was found in more than $80 \%$ (over the age of 20 years) in all populations. These data suggested that allelic loss of PTCH1 has different consequences in different genetic backgrounds and in different tissues. The analysis of molecules involved in these differences will help to prevent or delay the onset of BCCs.

\section{CONFLICT OF INTEREST}

The authors declare no conflict of interest.

\section{ACKNOWLEDGEMENTS}

We thank the patients and their families for making this study possible. This work was supported, in part, by the Research fund of the Hyogo College of Medicine.

1 Gorlin, R. J. Nevoid basal cell carcinoma (Gorlin) syndrome: unanswered issues. J. Lab. Clin. Med. 134, 551-552 (1999).

2 Cohen, Jr M. M. Nevoid basal cell carcinoma syndrome: molecular biology and new hypotheses. Int. J. Oral. Maxillofac. Surg. 28, 216-223 (1999).

3 Kimonis, V. E., Goldstein, A. M., Pastakia, B., Yang, M. L., Kase, R., DiGiovanna, J. J. et al. Clinical manifestations in 105 persons with nevoid basal cell carcinoma syndrome. Am. J. Med. Genet. 69, 299-308 (1997).

4 Johnson, R. L., Rothman, A. L., Xie, J., Goodrich, L. V., Bare, J. W., Bonifas, J. M. et al. Human homolog of patched, a candidate gene for the basal cell nevus syndrome. Science 272, 1668-1671 (1996).

5 Hahn, H., Wicking, C., Zaphiropoulous, P. G., Gailani, M. R., Shanley, S., Chidambaram, A. et al. Mutations of the human homolog of Drosophila patched in the nevoid basal cell carcinoma syndrome. Cell 85, 841-851 (1996).

6 Unden, A. B., Holmberg, E., Lundh-Rozell, B., Stähle-Bäckdahl, M., Zaphiropoulos, P. G., Toftgård, R. et al. I. Mutations in the human homologue of Drosophila patched (PTCH) in basal cell carcinomas and the Gorlin syndrome: different in vivo mechanisms of PTCH inactivation. Cancer Res. 56, $4562-4565$ (1996).

7 Stone, D. M., Hynes, M., Armanini, M., Swanson, T. A., Gu, Q., Johnson, R. L. et al. The tumour-suppressor gene patched encodes a candidate receptor for Sonic hedgehog. Nature 384, 129-134 (1996).

8 Marigo, V., Davey, R. A., Zuo, Y., Cunningham, J. M. \& Tabin, C. J. Biochemical evidence that patched is the Hedgehog receptor. Nature 384, 176-179 (1996).

9 Lindström, E., Shimokawa, T., Toftgård, R. \& Zaphiropoulos, P. G. PTCH mutations: distribution and analyses. Hum. Mutat. 27, 215-219 (2006).

10 Wolter, M., Reifenberger, J., Sommer, C., Ruzicka, T. \& Reifenberger, G. Mutations in the human homologue of the Drosophila segment polarity gene patched (PTCH) in 
sporadic basal cell carcinomas of the skin and primitive neuroectodermal tumors of the central nervous system. Cancer Res. 57, 2581-2585 (1997).

11 Xie, J., Johnson, R. L., Zhang, X., Bare, J. W., Waldman, F. M., Cogen, P. H. et al. Mutations of the PATCHED gene in several types of sporadic extracutaneous tumors. Cancer Res. 57, 2369-2372 (1997).

12 Epstein, E. H. Basal cell carcinomas: attack of the hedgehog. Nat. Rev. Cancer 8, 743-754 (2008).

13 Teglund, S. \& Toftgard, R. Hedgehog beyond medulloblastoma and basal cell carcinoma. Biochim Biophys Acta 1805, 181-208 (2010).

14 Pan, S., Dong, Q., Sun, L. S. \& Li, T. J. Mechanisms of inactivation of PTCH1 gene in nevoid basal cell carcinoma syndrome: modification of the two-hit hypothesis. Clin. Cancer Res. 16, 442-450 (2010).

15 Takahashi, C., Kanazawa, N., Yoshikawa, Y., Yoshikawa, R., Saitoh, Y., Chiyo, H. et al. Germline PTCH1 mutations in Japanese basal cell nevus syndrome patients. J. Hum. Genet. 54, 403-408 (2009).

16 Barreto, D. C., Gomez, R. S., Bale, A. E., Boson, W. L. \& De Marco, L. PTCH gene mutations in odontogenic keratocysts. J. Dent. Res. 79, 1418-1422 (2000).

17 Minami, M., Urano, Y., Ishigami, T., Tsuda, H., Kusaka, J. \& Arase, S. Germline mutations of the PTCH gene in Japanese patients with nevoid basal cell carcinoma syndrome. J. Dermatol. Sci. 27, 21-26 (2001).

18 Ogata, K., Ikeda, M., Miyoshi, K., Yamamoto, Y., Yamamoto, T., Osaki, T. et al. Naevoid basal cell carcinoma syndrome with a palmar epidermoid cyst, milia and maxillary cysts. Br. J. Dermatol. 145, 508-509 (2001).

19 Fujii, K., Kohno, Y., Sugita, K., Nakamura, M., Moroi, Y., Urabe, K. et al. Mutations in the human homologue of Drosophila patched in Japanese nevoid basal cell carcinoma syndrome patients. Hum. Mutat. 21, 451-452 (2003).

20 Tate, G., Li, M., Suzuki, T. \& Mitsuya, T. A new germline mutation of the PTCH gene in a Japanese patient with nevoid basal cell carcinoma syndrome associated with meningioma. Jpn. J. Clin. Oncol. 33, 47-50 (2003).

21 Nagao, K., Togawa, N., Fujii, K., Uchikawa, H., Kohno, Y., Yamada, M. et al. Detecting tissue-specific alternative splicing and disease-associated aberrant splicing of the PTCH gene with exon junction microarrays. Hum. Mol. Genet. 14, 3379-3388 (2005).

22 Tanioka, M., Takahashi, K., Kawabata, T., Kosugi, S., Murakami, K., Miyachi, Y. et al. Germline mutations of the PTCH gene in Japanese patients with nevoid basal cell carcinoma syndrome. Arch. Dermatol. Res. 296, 303-308 (2005).
23 Fujii, K., Ishikawa, S., Uchikawa, H., Komura, D., Shapero, M. H., Shen, F. et al. High-density oligonucleotide array with sub-kilobase resolution reveals breakpoint information of submicroscopic deletions in nevoid basal cell carcinoma syndrome. Hum. Genet. 122, 459-466 (2007)

24 Tachi, N., Fujii, K., Kimura, M., Seki, K., Hirakai, M. \& Miyashita, T. New mutation of the PTCH gene in nevoid basal-cell carcinoma syndrome with West syndrome. Pediatr. Neurol. 37, 363-365 (2007).

25 Honma, M., Ohishi, Y., Uehara, J., Ibe, M., Kinouchi, M., Ishida-Yamamoto, A. et al. A novel PTCH1 mutation in a patient of nevoid basal cell carcinoma syndrome. J. Dermatol. Sci. 50, 73-75 (2008).

26 Otsubo, S., Honma, M., Asano, K., Takahashi, H. \& lizuka, H. A novel germ-line mutation of PTCH1 gene in a Japanese family of nevoid basal cell carcinoma syndrome: are the palmoplantar pits associated with true basal cell carcinoma? J. Dermatol. Sci. 51, 144-146 (2008).

27 Sasaki, R., Saito, K., Watanabe, Y., Takayama, Y., Fujii, K., Agawa, K. et al. Nevoid basal cell carcinoma syndrome with cleft lip and palate associated with the novel PTCH gene mutations. J. Hum. Genet. 54, 398-402 (2009).

28 Sasaki, R., Miyashita, T., Matsumoto, N., Fujii, K., Saito, K. \& Ando, T. Multiple keratocystic odontogenic tumors associated with nevoid basal cell carcinoma syndrome having distinct PTCH1 mutations: a case report. Oral Surg. Oral Med. Oral Pathol. Oral Radiol. Endod. 110, e41-e46 (2010).

29 Evans, D. G., Ladusans, E. J., Rimmer, S., Burnell, L. D., Thakker, N. \& Farndon, P. A. Complications of the naevoid basal cell carcinoma syndrome: results of a population based study. J. Med. Genet. 30, 460-464 (1993).

30 Shanley, S., Ratcliffe, J., Hockey, A., Haan, E., Oley, C., Ravine, D. et al. Nevoid basal cell carcinoma syndrome: review of 118 affected individuals. Am. J. Med. Genet. 50, 282-290 (1994).

31 Lo Muzio, L., Nocini, P. F., Savoia, A., Consolo, U., Procaccini, M., Zelante, L. et al. Nevoid basal cell carcinoma syndrome. Clinical findings in 37 Italian affected individuals. Clin. Genet. 55, 34-40 (1999).

32 Ahn, S. G., Lim, Y. S., Kim, D. K., Kim, S. G., Lee, S. H. \& Yoon, J. H. Nevoid basal cell carcinoma syndrome: a retrospective analysis of 33 affected Korean individuals. Int $J$ Oral Maxillofac Surg. 33, 458-462 (2004).

33 Habibi, A. \& Jafarzadeh, H. Nevoid basal cell carcinoma syndrome: a 17-year study of 19 cases in Iranian population (1991-2008). J. Oral Pathol. Med. 39, 677-680 (2010). 